\title{
Experimental Study on Unintended Needle Dislodgement Detection Circuit Using a Non-invasive-type Sensor
}

\author{
Naofumi Nakaya ${ }^{a,}{ }^{,}$, Satoshi Watanabe ${ }^{\mathrm{b}}$, Yukio Mori ${ }^{\mathrm{c}}$, Naruki Shirahama ${ }^{\mathrm{d}}$, Takayuki Abe ${ }^{\mathrm{e}}$, Kazuo Aoki ${ }^{\mathrm{f}}$ \\ ${ }^{a}$ Faculty of Health Sciences, Tsukuba International University, 6-20-1 Manabe Tsuchiura, Ibaraki 300-0051, Japan \\ ${ }^{\mathrm{b}}$ School of Health Sciences at Odawara, International University of Health and Welfare, 1-2-25 Shiroyama Odawara, \\ Kanagawa 250-8588, Japan \\ ${ }^{\mathrm{c}}$ Department of Mechanical and Electronic Engineering, Salesian Polytechnic, 4-6-8 Oyamagaoka Machida, Tokyo \\ 194-0215, Japan \\ ${ }^{\mathrm{d}}$ Department of Creative Engineering, National Institute of Technology, Kitakyushu College, 5-20-1 Kokuraminami-ku, \\ Kitakyushu-shi, Fukuoka 802-0985, Japan \\ 'Department of Clinical Engineering, Tokyo Women's Medical University, 8-1 Kawada-cho Shinjuku-ku, Tokyo \\ 162-8666, Japan \\ ${ }^{\mathrm{f}}$ College of Science and Technology, Nihon University, 1-8-14 Kandasurugadai Chiyoda-ku, Tokyo 101-8308, Japan \\ *Corresponding Author: n-nakaya@tius.ac.jp
}

\begin{abstract}
Introduction: Accidental needle dislodgement during blood purification therapy has emerged as a problem, which occasionally results in fatalities. In this study, we examined such a circuit with an aim to detect the indwelling needle dislodgement.

Method: Our circuit uses electrostatic capacitive coupling that is electrically connected to the dialysis circuit and to the patient's body; hence, it does not require the direct contact of electrodes with blood (non-invasive-type sensor). Indwelling needle dislodgement was detected according to the variations in impedance between various electrodes. We examined the circuit characteristics under different experimental conditions.

Results and Discussion: In the experimental environment, we obtained the optimum values of signal frequency $(10 \mathrm{kHz})$ and load resistor $(200 \mathrm{k} \Omega)$. Additionally, the characteristics of the circuit were obtained under various conditions. Thus, our circuit may improve the safety of blood purification therapy.
\end{abstract}

Keywords: Blood purification, Non-invasive type sensor, Unintended indwelling needle dislodgement.

\section{Introduction}

In Japan, 320,000 chronic dialysis therapies have been performed so far, with approximately 6,000 patients increasing annually ${ }^{(1)}$. Dialysis is performed on patients to sustain life after impaired kidney function, which can only be revived otherwise by renal transplantation. However, as per the latest statistical survey conducted during January 1 to December 31, 2014, only 127 patients from among 12757 patients in Japan desiring renal transplantation could undergo renal transplantations ${ }^{(2)}$. Therefore, it is assumed that the majority of these unattended patients seek continued or fresh hemodialysis because it becomes critical to maintain their life. Hemodialysis is performed by extracorporeal circulation of blood in a patient to eliminate the surplus moisture and wastes using an artificial kidney that can be referred to as a dialyzer, which is followed by returning the purified blood into the body. This procedure involves the risk of mass blood loss due to intentional withdrawal of the indwelling needle on the returning side. Such a needle accident is assumed to occur most frequently in case of dialysis medical accidents; sometimes, the life of a patient is also endangered ${ }^{(3)}$.

To prevent a needle accident, two major categories of countermeasures are introduced to the human and machine sides; the dialysis machine, which represents the machine side, is incapable of detecting needle dislodgement. This situation relies on a method that detects the decrease in the returning side pressure and a method of circulation monitoring by the medical staff and ingenuity of the dialysis circuit fixation method on the human side. 
However, the detection method based on the pressure difference on the blood return side is dependent on the resistance by the needle capillary, which is assumed to be influenced by a small difference in the blood vessel pressure; further, the detection is also difficult ${ }^{(4)}$. Additionally, as stated above, even with countermeasures on the human side, it is currently deemed to be impossible to effectively prevent a needle accident from being the major reason for medical accidents during dialysis.

Therefore, in this study, we develop a circuit that senses unexpected needle extraction using a sensing electrode that does not require direct contact with blood (non-invasive-type sensor) and using high-frequency signals to capacitively couple the blood circuit and the human body. Additionally, we evaluated the characteristics of the circuit in this study.

\section{Method}

\subsection{Structure of the needle dislodgement detection circuit}

Figure 1 illustrates the needle dislodgement detection circuit.

The dialysis circuit comprises a dialysis monitoring device (DCS-26: Nikkiso, Tokyo, Japan), a blood circuit for artificial kidney (H-702-TQB: Toray, Tokyo, Japan), an indwelling needle for dialysis (Happy Cass V, 15-17 G: Medikit, Tokyo, Japan), and a dialyzer (FB-210 UßGA: Nipro, Osaka, Japan).

The needle dislodgement detection circuit includes a clip-shaped detection electrode, a load resistor, an instrumentation amplifier (INA 128; Texas Instruments, Dallas, Texas, United States), a signal input transformer(MG-21; Tamura, Tokyo, Japan), a detection electrode transformer (MG-12; Tamura), and a function generator (33521A; Agilent Technology, Santa Clara, California, United States).

The detection electrode is attached to the arterial side (blood collection side) of the blood circuit and the venous side (blood return side) at a certain interval from the tip of the needle, and it is connected to the function generator via load resistor. Voltage drop due to the flow of current in the load resistor is amplified by the amplifier, which is further rectified and finally smoothed so that it can be observed as a direct current voltage (detection voltage).

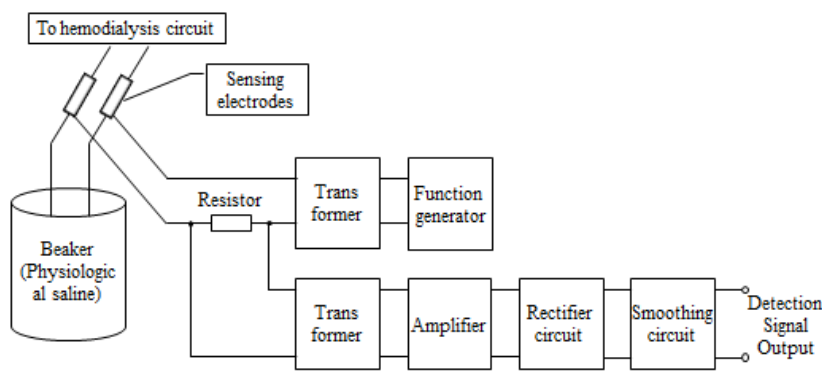

Fig. 1. The needle dislodgement detection circuit

When the needle tip is immersed in the physiological saline solution of a beaker, which is similar to that in a human body, the electrode and beaker are electrostatically capacitively coupled, and a very small amount of current flows. However, when the blood circuit is disconnected, the electrical characteristics of the needle tip changes, and the voltage value at both the ends of the resistance decreases. Applying this change enables the detection of a needle dislodgement.

\subsection{Principle of the detection electrode}

The dialysis circuit comprises an electrical insulator (polyvinyl chloride) with an extremely high electric resistance ( $\mathrm{M} \Omega$ order). Because electric capacity is dependent on the blood circulation, the introduction of air into the circuit after needle dislodgement decreases the electric capacity, which reflects the low dielectric constant of air (approximately 1) relative to that of the blood (approximately 80).

As depicted in Figure 2, the detection electrodes were attached to the arterial (blood collection) and venous (blood return) sides of the blood circuit at two different positions. Hence, the electrodes were connected to the patient via an electrical insulator such as the blood circuit or dialysis indwelling needle. Accordingly, an electrical equivalent circuit (Fig. 3) was formed between the two detection electrodes. In this circuit, $R$ represents an extremely large impedance component that is introduced due to the human body and blood circuit, and $C$ represents the capacitance component because of the electrostatic capacitance between the electrodes. Because these electrodes were connected in parallel, less current flowed into the impedance component; therefore, the capacitance component was observed to dominate the current value.

Capacitive reactance can be expressed using the following equation: 


$$
X_{C}=\frac{1}{j \omega C}=-j \frac{1}{\omega C}[\Omega]
$$

Using Equation (1), we can observe that capacitive reactance exhibits low electrical impedance when an alternating current (AC) signal is considered to be the input. Therefore, when a weak AC $(<7 \mu \mathrm{A})$ signal that does not affect the human body output by the proposed circuit is applied to the detection electrode, only a small amount of current flows through capacitive electrical coupling. However, when one side of the indwelling needle is disconnected, the dielectric material existing between the electrodes is replaced with a model wherein part of the air is sandwiched by the blood. Additionally, the electrostatic capacity of a single dielectric capacitor using a metal parallel plate is obtained by setting the area of the electrode plate to $S\left[\mathrm{~m}^{2}\right]$, the dielectric constant, $\varepsilon_{0}$, in vacuum, and the relative dielectric constant, $\varepsilon_{\mathrm{s}}$, of the substance that exists between the electrodes. Assuming a model in which a dielectric material with a thickness of $l[\mathrm{~m}]$ is inserted, the electrostatic capacity can be expressed by the following equation:

$$
C=\frac{\varepsilon S}{l}=\frac{\varepsilon_{0} \varepsilon_{S} S}{l}[\mathrm{~F}]
$$

At this time, if the dielectric material is partially replaced with air and if the dielectric constant of the original dielectric is $\varepsilon_{1}$, the dielectric constant of air is $\varepsilon_{2}$, and the thicknesses are $l_{1}$ and $l_{2}[\mathrm{~m}]$, as depicted in Equation (3). As can be observed from this equation, the value of electrostatic capacity, $C$, becomes smaller than that in Equation (2).

$$
C=\frac{S}{\frac{l_{1}}{\varepsilon_{1}}+\frac{l_{2}}{\varepsilon_{2}}}[\mathrm{~F}]
$$

As the electrostatic capacity, $C$, decreases, the capacitive reactance increases, which is in accordance with Equation (1), and the electrical impedance between the electrodes also increases. Due to impedance variation, the voltage drop at the load resistor (Fig. 3) decreases; therefore, the detection signal output decreases. By monitoring the detection signal output, the proposed circuit enables the detection of the needle tip.

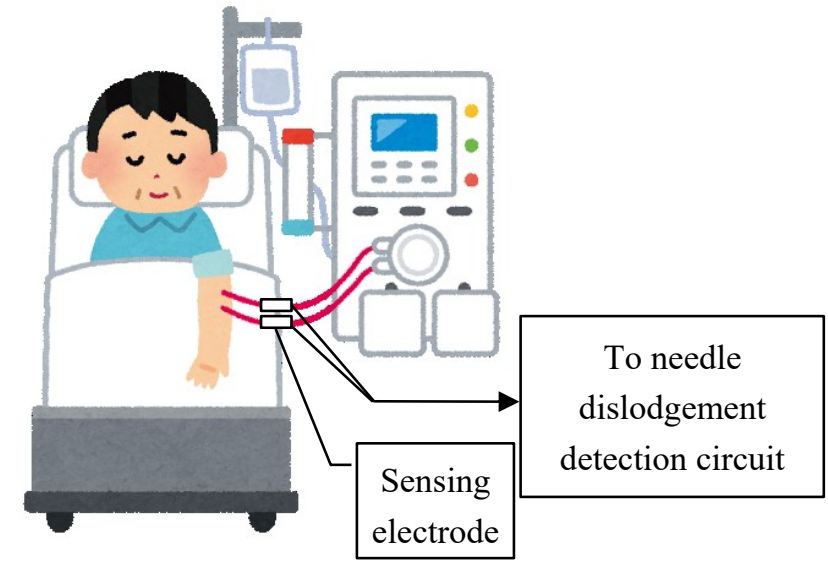

Fig. 2. Schematic of sensor attachment to the dialysis circuit $^{(5)}$

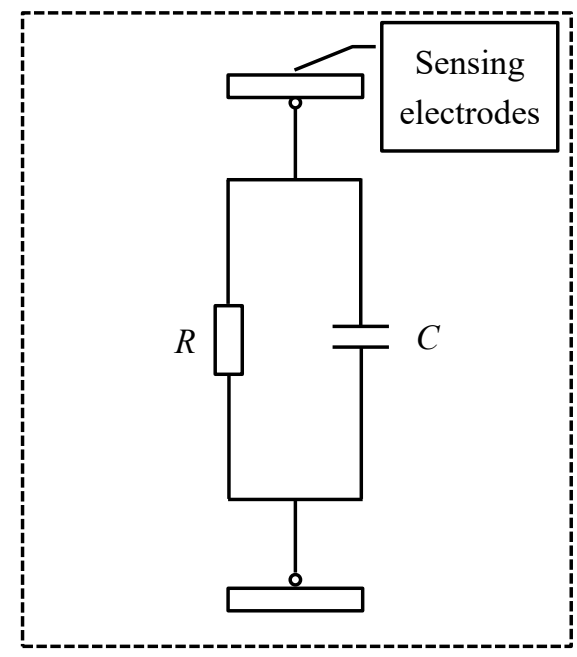

Fig. 3. Electrical equivalent circuit $^{(5)}$

\subsection{Experimental method}

Using the needle dislodgement detection circuit and indwelling needle $(17 \mathrm{G})$ depicted in Figure 1, the state in which the needle was immersed at both sides was defined as normal; further, when one side of the needle was disconnected, the state was defined as the needle dislodgement state for which a high-frequency signal (sine wave, $10 \mathrm{kHz}, 10 \mathrm{~V}_{\mathrm{p}-\mathrm{p}}$ ) was applied. The blood pump was operated at $210 \mathrm{~mL} / \mathrm{min}$ for circulation. The detection electrode was mounted at a position that was $30 \mathrm{~cm}$ away from the tip of the blood circuit, and the electrode area was set to be $25 \mathrm{~cm}^{2}$. Furthermore, the gain of an instrumentation amplifier (hereinafter referred to as an amplifier) was adjusted so that the detection voltage was $2.5 \mathrm{~V}$ in the normal state. The measurements were set according to the following two points: 
i) as the reference value for the gain, the input voltage to the amplifier in a normal state was observed using a digital multimeter (U3401A; Keysight Technologies, Santa Rosa, California, United States ) and

ii) the difference in the detection voltages of the normal and needle was observed using a digital multimeter.

Using the aforementioned conditions as the basic conditions, the experiment was performed under the following conditions:

The electrode distance was $30-60 \mathrm{~cm}$ from the blood circuit tip, and the electrode area was $15-45 \mathrm{~cm}^{2}$. However, the short-axis direction was fixed as $5 \mathrm{~cm}$, and it was changed in the long-axis direction to $3-9 \mathrm{~cm}$, having the signal frequency ( $1 \mathrm{kHz}$ to $1 \mathrm{MHz}$ ), load resistor (10 $\Omega$ to 1 $\mathrm{M} \Omega$ ), blood pump flow rate $(10-300 \mathrm{~mL} / \mathrm{min})$, and gauge of indwelling needle (direct connection, outer diameter of approximately $4.1 \mathrm{~mm}, 15-17 \mathrm{G})$.

\section{Results and Discussion}

Figures 4-9 illustrate the experimental results of each condition.

The input voltage to the amplifier tended to increase as the signal frequency increased up to $100 \mathrm{kHz}$. However, it tended to decrease at $>100 \mathrm{kHz}$. Additionally, the difference in the detected voltage tended to decrease at a frequency of $>10 \mathrm{kHz}$. When the signal frequency was 10 $\mathrm{kHz}$, the difference in the detected voltage was approximately $10 \%$ (Fig. 4).

The input voltage to the amplifier tended to become high as the load resistor increased. However, almost no change was noted in the difference in detected voltage due to a load resistor of up to $200 \mathrm{k} \Omega$, which indicated a decreasing tendency at $>200 \mathrm{k} \Omega$ (Fig. 5).

A strong negative correlation was observed in the difference in detection voltage with respect to the electrode distance from the tip of the dialysis circuit (Fig. 6).

A strong positive correlation was noted between the detection electrode area and the difference between the detection voltages (Fig. 7).

No correlation was recorded between the blood pump flow rate and the detected voltage (Fig. 8).

A strong positive correlation was noted between the gauge of the indwelling needle and the difference in the detected voltage (Fig. 9).

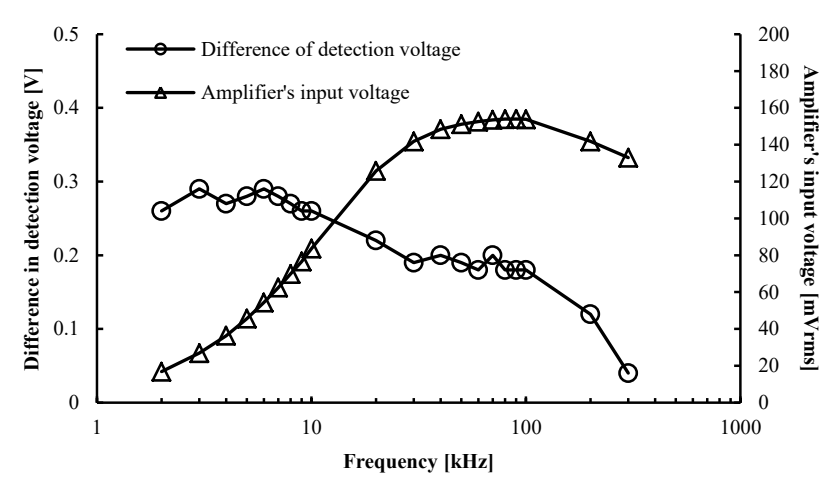

Fig. 4. Result of the frequency-characteristic experiment

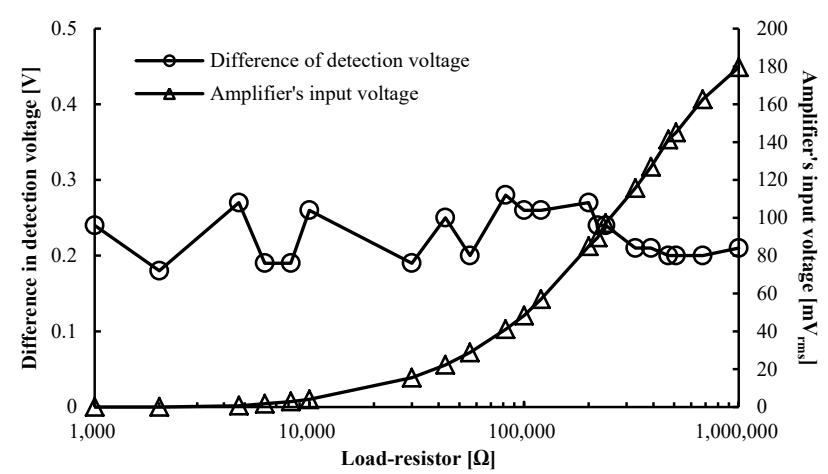

Fig. 5. Result of the load-resistor characteristic experiment

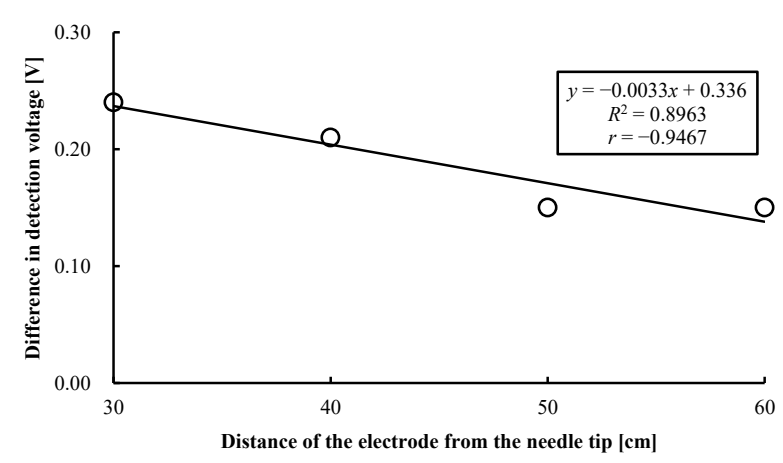

Fig. 6. Result of the electrode-distance characteristic experiment 


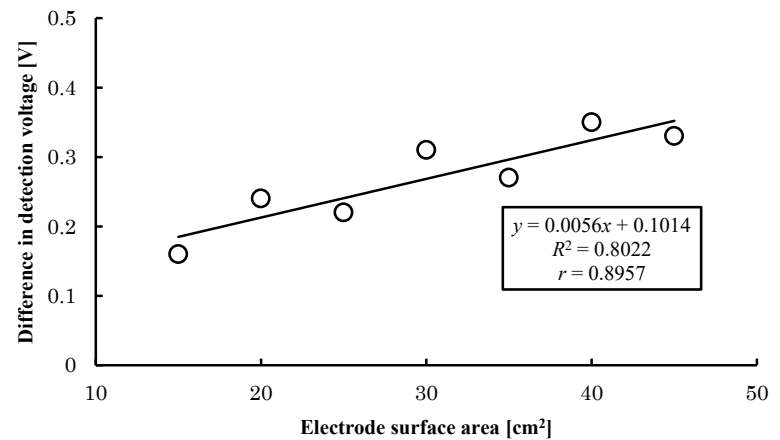

Fig. 7. Result of the electrode-area characteristic experiment

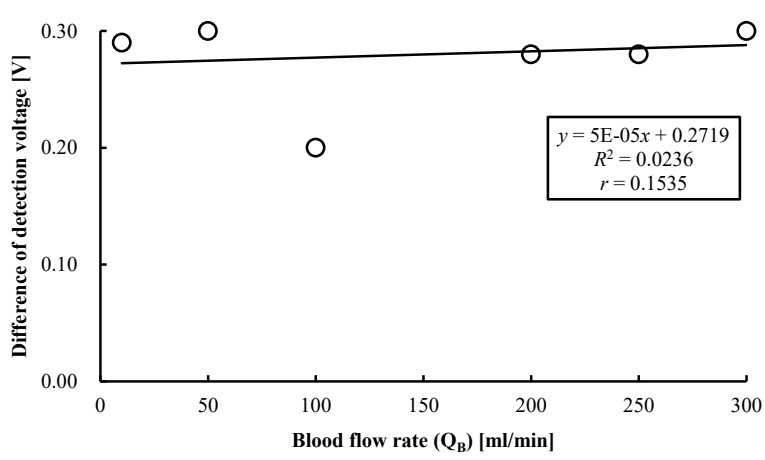

Fig. 8. Results of the blood pump flow-dependence experiment

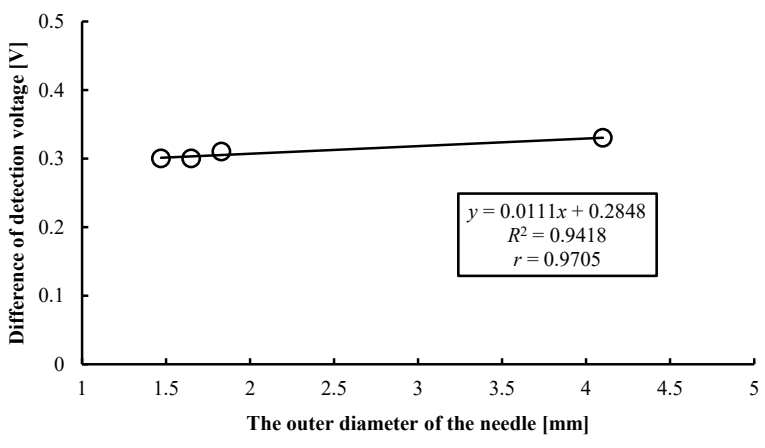

Fig. 9. Results of the indwelling needle gauge-dependency experiment

Using the results of the frequency-characteristic experiment (Fig. 4), it can be observed that, as the signal frequency increases, the input voltage of the amplifier tends to increase with the peak obtained at $100 \mathrm{kHz}$. Therefore, when the signal frequency is $100 \mathrm{kHz}$, the gain may be minimized. However, to detect unexpected needle removal from a patient, a large detected voltage difference is useful to prevent malfunction. From the viewpoint of detection voltage difference, the peak was obtained at $10 \mathrm{kHz}$; therefore, a decreasing trend was observed. Accordingly, the signal frequency of $10 \mathrm{kHz}$ seemed to be reasonable.

Based on the results of the resistance characteristic experiment (Fig. 5), the input voltage of the amplifier tended to increase with increasing load resistor. However, because the difference in the detected voltage exhibited a decreasing tendency at $>200 \mathrm{k} \Omega$, the load resistor seemed to be adequate for $200 \mathrm{k} \Omega$.

Based on the result of the detection electrode-distance characteristic experiment (Fig. 6), a strong negative correlation was recognized. Accordingly, it was assumed that, from the viewpoint of electrical characteristics, the detection electrode should be mounted near the tip of the probe tip as much as possible. Furthermore, it seems important to investigate the maximum distance that can be detected because the attachment part can be considered to be a factor of increasing discomfort due to contact with the dialysis circuit fixing part of the patient.

Based on the result of the detection electrode-area characteristic experiment (Fig. 7), a strong positive correlation was recognized. Accordingly, it was assumed that the electrode area should be maximized as much as possible from the viewpoint of electrical characteristics. However, for dialysis, it seems necessary to study the minimum detectable area as it has become impossible to bend the circuit, which can become a factor for increasing discomfort due to contact with the patient.

The results of the blood pump flow rate-dependence experiment given in Figure 8 indicate no correlation because the fitting of the approximate expression was poor $\left(R^{2}=0.0236\right)$. Therefore, it was considered that the treatment condition barely influenced the blood pump flow rate.

The results of the indwelling needle gauge-dependency experiment (Fig. 9) exhibited a strong positive correlation. Considering the results obtained in this study, it seems necessary to examine the extracted needle detection system because the rate of decrease in the detected voltage differs with different gauge size of the indwelling needle and the treatment condition such as the direct connection to the catheter.

\section{Conclusions}

In this research, the basic characteristics of the extracted needle detection circuit were examined to expand our understanding of the prevention of medical accidents due to unintentional needle extraction. 
Our results helped to achieve the following conclusions:

i) the signal frequency added to the detection electrode was considered to be reasonable at 10 $\mathrm{kHz}$;

ii) a $200-\mathrm{k} \Omega$ load resistor was considered to be reasonable;

iii) the electrode distance from the needle tip was strongly and negatively correlated with the detection voltage;

iv) the detection electrode area was strongly and positively correlated to the detection voltage;

v) no blood pump flow rate dependence was observed;

vi) the gauge of the indwelling needle was positively correlated with the detection voltage in a strong manner;

vii) the detected current was extremely weak, and it was assumed that the criteria prescribed in JIS T 0601-1 would be sufficiently cleared; and

viii) in future, the difference between the electrical characteristics of beakers and human patients should be considered.

The experiment based on the basic conditions by the extracted needle detection circuit proposed in this research revealed that the difference between the detection voltage at the time of normal operation and the detection voltage at the time of needle discharge abnormality was approximately $10 \%$. Therefore, using a microcomputer board, automatic detection of a needle during blood purification therapy can be performed.

This study is limited by the use of beakers instead of the human body to perform the experiment because the electrical characteristics of the human body are different from that of a beaker. Therefore, in future, it is important to clear the ethical issues and to conduct clinical experiments in human and animal subjects to improve the relevance of the results.

\section{Acknowledgment}

We are particularly grateful for the assistance provided to us by the following graduates from Tokyo Electronics College, Eri Suzuki, Akino Yoshida, Shogo Sekikawa, Makoto Nakamura, Keita Sato, and Kenji Matsumae, and for the help from technical assistants.
This work was supported by the JSPS KAKENHI Grant Number JP17K15869.

\section{References}

(1) Patient Registration Committee, Japanese Society for Dialysis Therapy, Tokyo, Japan: "Current state of Japan's chronic dialysis therapy (December 31, 2014 currently)," http://docs.jsdt.or.jp/overview/, 2015 (in japanese)

(2) Japan organ transplant network, Tokyo, Japan: "Number of kidney provided and transplant by prefecture,"

http://www.jotnw.or.jp/file_lib/pc/datafile_offer_pdf/ki dneys_region2014.pdf, 2014 (in japanese)

(3) Yoshihei Hirasawa, et al.: "Study on the survey on actual condition of dialysis medical accident and formulation of accident countermeasure manual", Journal of Japanese Society for Dialysis Therapy, Vol.34, No.9, pp. 1257-1286, 2001 (in Japanese)

(4) Kumo Ichiro: "Apparatus and method for monitoring autotransfusion, transfusion or infusion," Patent of Japan, JP2008086581A, 2008

(5) Naofumi Nakaya, et al.: "Automatic Detection of Unintended Indwelling Needle Dislodgement," Proceedings of the 5th IIAE International Conference on Intelligent Systems and Image Processing 2017, pp.335-338, 2017 